\title{
CADASTRAL POSITIONING ACCURACY IMPROVEMENT: A CASE STUDY IN MALAYSIA
}

\author{
N. M. Hashim ${ }^{a^{*}}$, A. H. Omar ${ }^{a^{*}}$, K.M. Omar ${ }^{\text {a }}$, N. M. Abdullah ${ }^{a}$, M. H. M. Yatim ${ }^{a}{ }^{*}$ \\ ${ }^{a}$ Dept. of Geomatic Engineering, University Technology Malaysia, Skudai, Johor Bahru, MALAYSIA - \\ *norshahrizan@perlis.uitm.edu.my,*abdullahhisham@utm.my, kamaludinomar@utm.my, nazirah1976@gmail.com, \\ hafizyatim3005@gmail.com
}

KEY WORDS: Positional Accuracy Improvement, Legacy Dataset; Cadastral Modernization

\begin{abstract}
:
Cadastral map is a parcel-based information which is specifically designed to define the limitation of boundaries. In Malaysia, the cadastral map is under authority of the Department of Surveying and Mapping Malaysia (DSMM). With the growth of spatial based technology especially Geographical Information System (GIS), DSMM decided to modernize and reform its cadastral legacy datasets by generating an accurate digital based representation of cadastral parcels. These legacy databases usually are derived from paper parcel maps known as certified plan. The cadastral modernization will result in the new cadastral database no longer being based on single and static parcel paper maps, but on a global digital map. Despite the strict process of the cadastral modernization, this reform has raised unexpected queries that remain essential to be addressed. The main focus of this study is to review the issues that have been generated by this transition. The transformed cadastral database should be additionally treated to minimize inherent errors and to fit them to the new satellite based coordinate system with high positional accuracy. This review result will be applied as a foundation for investigation to study the systematic and effectiveness method for Positional Accuracy Improvement (PAI) in cadastral database modernization.
\end{abstract}

\section{INTRODUCTION}

Nowadays, many spatial datasets were formerly digitized from paper maps and subsequently updated. This means that the legacy datasets in use today are an amalgamation of data from different sources, combined at different times by different methodologies. These legacy datasets have relatively low positional accuracy caused by errors resulting from the production and measurement method employed according to the technological and legal changes over time (Sisman, 2014). In addition, the general causes of error in digitizing process such as distortion of source map, digitizing operational errors and ground coordinate system which possibly is constituted by a combination of systematic and random errors (Tong, Shi, \& Liu, 2009). With the change from hardcopy to digital datasets the needs for combine spatial data from different sources has dramatically increased. This process is crucial to allow different datasets to be jointly presented and analysed. The spatial integration of data from various sources requires an understanding about the positional accuracies of the geometries in the datasets to avoid mismatches and misinterpretations.

In line with the cadastral 2014 campaign, it has been recognized the value of highly accurate digital cadastral data and researchers globally are developing methods to enhance and improve the accuracy of the cadastral database. As a result, many countries around the world is modernizing the cadastral database from legacy cadastre or relative cadastre to accurate coordinate based cadastre known as Positional Accuracy Improvement (PAI) (Donnelly \& Hannah, 2006; Yaron A. Felus, 2007; Fradkin \& Doytsher, 2002; Hesse, Benwell, \&
Williamson, 1990; Hope, Gordini, \& Kealy, 2008; Klebanov \& Doytsher, 2009; Morgenstern, Prell, \& Riemer, 1989; Tamin, 1995). PAI is classified as a process of improving the position of the geometry coordinates of a feature in a geospatial dataset to better reflect its actual position (Rönsdorf, 2008). The PAI is defined as the refinement of low accurate legacy dataset by referring to more accurate dataset which guides realignment of the existing version of the dataset. Figure 1 illustrate the concept of PAI that have been discussed before.

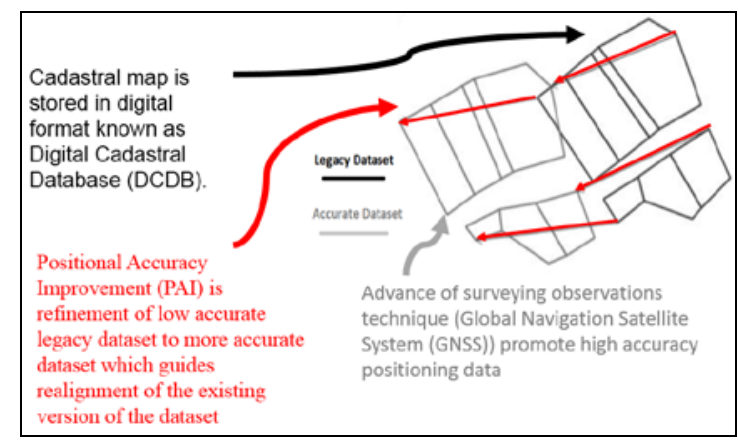

Figure 1. PAI concept

PAI is commonly applied in two situations, PAI of Reference Data and PAI of User Data (Rönsdorf, 2008). The PAI of Reference Data links with improving the position of geometries in a reference dataset that describes physical or abstract features of the earth. The features position relate to the absolute position in a standard Coordinate Reference System such as in case of Malaysia is Geocentric Datum of Malaysia 2000 (GDM 2000) 
or WGS-84 in global coordinate system. Meanwhile, the PAI of User Data describes the successive synchronization of legacy datasets with the already positionally improved reference dataset in order to remain the relationships between geometries. Based on the importance of PAI, the focus of this paper is to investigate and propose the positional accuracy improvement (PAI) method so that the future integration process towards accurate dataset can be improved and sustained. The detail discussion on PAI is explained in the following section.

\section{POSITIONAL ACCURACY IPROVEMENT}

The emergence of spatial based technology like Geographical Information System (GIS) as a main tool in spatial management results in an urgent need to maintain the spatial data in digital format. Nowadays, many countries around the world have recognized and appreciate the value of accurate digital cadastral database. Accurate, efficient and updated cadastral database offer the better basis for planning and implementation of variety of real estate application (Durgin, 1993; Effenberg, Enemark, \& Williamson, 1999; Ting \& Williamson, 1999). Several basic methods are possible for upgrading the legacy cadastral datasets such as resurvey all the cadastral parcel, reprocessing the existing survey data and upgrade of the existing cadastral datasets.

Resurvey all cadastral parcel possibly is the best technique to solve the problem, however that is the drastic solution. For this purpose, it would be necessary to reestablish the boundaries according to the new control framework or coordinate system. This process constitutes a tremendous effort and estimated cost is very high (Arvanitis \& Koukopoulou, 1999). Another solution is using the original observations based on the field book. According to the Buyong and Kuhn (1990) and Durgin (1993), maintaining the old measurement and incorporating new measurements might be accomplished by creating a measurement based multipurpose cadastral system.

However, based on the study that have been carried out in Israel by Perelmuter and Steinberg (1992), calculating the field book in reprocessing the cadastral database is inapplicable due to weakness of the original control network (datum), the updating in the field were marked in field sheet but were not recorded in the field book and missing of the field books. In addition, from the economic perspective, 20000 existing field book require hundreds of operators and take many years to accomplish (Fradkin \& Doytsher, 2002).

A potential alternative for restoring the legacy datasets with reasonable cost is the PAI process where the legacy dataset is transformed into new high accuracy dataset. The drastic changing and development of satellite based technology in data observation such as Global Navigation Satellite System (GNSS) also make the great possibility of PAI process in transforming the legacy datasets. Furthermore, the availability of high resolution aerial imagery and the popularity of open source application such as Google Earth are leading to predominant usage of imagery as background underlays to spatial dataset (Hope et al., 2008). The imagery which has high quality spatial resolution and absolute accuracy like Quick Bird imagery can be used as a base map to check the discrepancies between the legacy datasets and the satellite imagery.

The PAI process in general covers several important issues such as transformation, geometrical fitting and adjustment process.
The following section discussed clearly the issues involved in the PAI process.

\subsection{Transformation Issues in PAI}

Specific consideration is given to the transformation process in the PAI studies and researchers around the world have studied the impact of transformation model in PAI studies. In general, there are two types of transformation model in PAI which consist of global transformation and local transformation models (Tong, Liang, Xu, \& Zhang, 2011). According to Kang (2002) the method of global transformation is suitable to be applied when a small number of control points are used to calculate the transformation parameter and there is a system error in the source map. Meanwhile, the transformation model of Helmert Similarity Transformation and affine transformation model are regularly used in the global transformation (Greenfeld, 1997; Mikhail \& Ackermann, 1982; Wang, Di, \& Li, 2005).

However, the local transformation models are usually based on a planar subdivision using Delaunay Triangulation. For example, using rubber-sheeting techniques, the entire area is first divided into triangles whose vertices are the matched node pairs, and simplicial coordinates based on the vertices of each triangle are then used to determine the transformation parameters for the points inside each triangle (Cobb et al., 1998; Doytsher \& Gelbman, 1995; Kang, 2002). Saalfeld (1988) described the local transformations preserved in the topological neighborhood structure.

As such, the local affine transformations maintain linearity and parallelism on each triangle; however, these continuous transformations are not differentiable at the edges of the triangles (Saalfeld, 1993). For instance, a straight linear feature that spans several triangles may experience considerable distortion because of the inconsistency of the transformation at the triangle vertices. Hence, these local methods, which distort the source dataset to fit the target dataset, might account for this issue when they are used to upgrade the spatial datasets in a global area, within which the geometric characteristics and spatial relationships should be essentially preserved (Hope et al., 2008; Hope, Kealy, \& Hunter, 2006; Tong, Shi, \& Liu, 2005).

In the study in PAI in Victoria Australia, an initial 4 parameters of Helmert Transformation is applied to the lower accuracy legacy dataset, so that it is will be aligned to the new coordinate system of survey data (Hope et al., 2008). Yaron A. Felus (2007) tested four transformation models which consist of Translation (2 parameters), Similarity (4 parameters), Affine (6 parameters) and Projective (8 parameters) in PAI. The result of the experiments show that similarity transformation is the most suitable model in the case study area. Meanwhile, Tong et al. (2011) have tested five transformations models Translation, Scale and Translation, Similarity, Affine and Second Order Polynomial Model. Based on the different suggestions and findings of transformation process in PAI method, this study will investigate several transformation model (Translation, Scale and Translation, Similarity Transformation \& Affine Transformation) that better fits in the case study area.

\subsection{Geometric Condition in PAI}

Since the legacy dataset are less accurate in positioning, the integration between legacy datasets and higher updated 
accuracy like GNSS is one of the most possible methods to improve the legacy datasets accuracy (Hope et al., 2008). However, in her study, it is found that by simply replacing a sample of legacy dataset with more accurate version will lead to a distortion of the neighbouring geometry (Figure 2). In addition, often the relative geometry of the legacy datasets is better than its absolute accuracy and the supposed spatial relationship or relative geometry between features must be preserved.

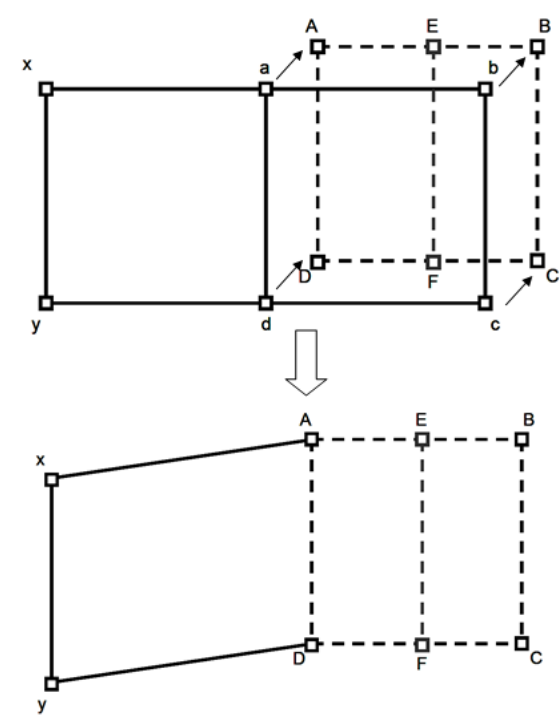

Figure 2. Cadastral boundaries (Solid Lines) and higher accuracy (Dashed Lines). Polygon xady is distorted if point abcd are simply replaced by ABCD (Hope et al., 2008).

Saalfeld (1988) proposed an iterative technique using rubber sheeting method when replacing the legacy dataset with higher accuracy dataset. In this study, the method developed aims to make the dataset consistent rather than improving the positional accuracy. Yaron A. Felus (2007) applied rubber sheeting method to stretch and locally transform the legacy datasets to fit with the new higher accuracy measurement. In this study, the existing ground monument should be surveyed and the coordinate must remain unchanged (fixed). The coordinate of the lost boundary were estimated from the parcel map and then were modified using rubber sheeting method.

Meanwhile, in the PAI process, the method of constrained least squares also classified as a well-established, rigorous technique for determining the optimal solution in geometrical fitting (Hope et al., 2008). Constrained least square possible to include geometric or topological constraints as observations in a least squares adjustment. For example, the use of rectangularities, collinearities and parallel lines to constrain cadastral adjustments has been demonstrated (Hesse et al., 1990; Merrit \& Masters, 1999), as has the preservation of areas (Tong et al., 2005).

\subsection{Adjustment Method in PAI}

To achieve an optimal solution in PAI, the method of least squares (LS) is often employed toward improving the positional accuracy of spatial datasets (Casado, 2006; Ghilani, 2010; Gielsdorf, Gruendig, \& Aschoff, 2004; Hope et al., 2008; Merrit \& Masters, 1999; Merritt, 2005; Tamin, 1995; Tong et al., 2009). The LS method is a well-established technique for solving an over determined system of equations by minimizing a weighted quadratic form of the residuals. Its application in estimating parameters in coordinate transformation can be found in the literature; for example, Wolf and Ghilani (2006) and Koch (2013). Tamin (1995) presented a methodology to create a digital cadastral overlay through upgrading digitized cadastral data.

Merrit and Masters (1999) and Merritt (2005) developed the spatial adjustment engine based on the least squares method and applied it to improve the accuracy of cadastral data in Australia. Tong et al. (2005) presented a least squares adjustment model to resolve inconsistencies between the digitized and registered areas of cadastral parcels, and further improved the adjustment model by introducing scale parameters to reduce the influence of systematic error in the adjustment (Tong et al. 2009).

Yaron A. Felus (2007) presented a workflow of three steps used to enhance the spatial accuracy of digital cadastral maps: a global transformation from an old local system to a GPS-based WGS-84 system; a rubber-sheeting transformation for modifying boundary corners to fit existing ground features; and a LS adjustment with stochastic constraints to include additional cadastral information and geometric conditions. Hope et al. (2008) proposed a method of least squares with inequalities for data integration, in which topological relationships are modelled in the form of inequalities and optimal positioning solutions are obtained while preserving the spatial relationships among features.

As clearly discussed, the legacy dataset positional accuracy has to be improved if they are to remain valuable in line with the requirement of spatial data advances of present. With the advancement of surveying observations techniques especially Global Navigation Satellite system (GNSS), PAI process is inevitable. Malaysia also participated in the trend of the PAI campaign and this has led to the Malaysia cadastral database development knowns as National Digital Cadastral Database (NDCDB).

\section{MALAYSIA CADASTRAL DATABASE REFORM}

Cadastral surveying in Malaysia stated more than one hundred years ago for supporting the land development activities. There have been many changes made officially in terms of operational, structural and institutional in Malaysia cadastral survey over the past decades regarding to the various factors especially quality assurance, efficiency, enhancement and technology updated.

DSMM stared the digital era of cadastral database with the execution of the Computer Assisted Land Survey System (CALS) pilot project in 1985 in Johor. This, subsequently, led to the implementation in the State of Pahang in 1986 and the implementation of Mini-CALS system in all remaining State's JUPEM of Peninsular Malaysia in 1992. (Desa, 2005; Mohd Yusoff \& Abdul Halim, 2012; Mohd Yusoff, Jamil, \& Abdul Halim, 2013; Omar, A Kadir, \& Sidek, 2006).

With the growth of Geographical Information System (GIS), DSMM move to create a Digital Cadastral Database (DCDB) as a foundation to encourage the development of GIS in the cadastral management (Mohd Yusoff \& Abdul Halim, 2012). Consequently, DSMM introduced the Cadastral Data Management System (CDMS) in the year 1998, principally to repopulate the DCDB in every State's JUPEM and allow 
multiple users to operate the user-limited Mini-CALS system and later the CDMS Upgrade is implemented to all DSMM states from November 2011.

As high expectancy relating to land delivery system increases, DSMM has embarked on a cadastral modernization program with the implementation of the eKadaster project. The eKadaster project led to the creation of a survey accurate database at the national level and usage of Coordinated Cadastral Systems (CCS). The modernization in the cadastral surveying system is inevitable to ensure that the cadastral surveys meet the desired accuracy required.

The eKadaster optimized the usage of ICT, GIS and survey technologies and made major modification in observation data adjustment from the traditional Bowditch and Transit methods to a Survey Accurate Coordinate system using Least Square Adjustment (Mohd Yusoff \& Abdul Halim, 2012). In the CCS era, the full utilization of Global Navigation Satellite System (GNSS) is applied and several amendment in survey regulation regarding the usage of GNSS is implemented. The CCS program is targeted to develop a homogeneous cadastral database known as National Digital Cadastral Database System (NDCDB) which is based on the geocentric datum with a spatial accuracy better than 5 centimetres in urban area and better than 10 centimetres in semi urban and rural areas.

NDCDB is a coordinate-based cadastral system with the coordinates being given legal significance. NDCDB applied the concept of the earth-cantered datum, a single projection system for the whole country and the application of least-square adjustment technique in the adjustment of estimate survey errors. To maximize and fully utilize the advantages of the NDCDB, it is important to understand the PAI process contributed to the success of the NDCDB development and sustainability. Based on the literature studies, this study aim to recommend a framework that uses a PAI to update the accuracy of cadastral dataset and it is explained in the next section.

\section{CADASTRAL MAP IMPROVEMENT}

The proposed PAI process of NDCDB concentrating on three main stages which consist of Initial Transformation, Geometrical Fitting and Positional Evaluation. The first step is using a global coordinate transformation, performed to convert the legacy datasets to a new coordinate system.

It is performed to convert the legacy dataset from the Old Cassini Coordinate System (Cassini-Soldner projection, with a datum MRT and Modified Everest reference ellipsoid to a new GPS-based coordinate system (Cassini-Soldner Projection with a datum GDM2000 with GRS80 reference ellipsoid. As such, $(\mathrm{X}, \mathrm{Y})$ are the surveyed coordinates of GCPs with higher positional accuracy, and that $(\mathrm{x}, \mathrm{y})$ are the legacy dataset coordinates, thus a general form of the initial transformation model in PAI adjustment is written as

$$
\begin{aligned}
& X=F x(x, y) \\
& Y=F y(x, y)
\end{aligned}
$$

where Fx and Fy are the transformation functions in the $\mathrm{x}$ - and $\mathrm{y}$-directions, respectively. In this study, by adopting similar method by Tong et al. (2011), four specific model will be tested in the transformation process.
Translation model

$$
\begin{aligned}
& X=a 0+x \\
& Y=b 0+y
\end{aligned}
$$

where $\mathrm{a} 0$ and $\mathrm{b} 0$ are the two unknown translation parameters. A minimum of one GCP is required to estimate the translation parameters.

\section{Scale and translation model}

$$
\begin{aligned}
& X=a 0+a 1 x \\
& Y=b 0+b 1 y
\end{aligned}
$$

where $\mathrm{a} 1$ and $\mathrm{b} 1$ are the two scale parameters in addition to the shift parameters in Equation (2). A minimum of two GCPs is required to estimate these four parameters in the model.

Similarity transformation model

$$
\begin{aligned}
& X=a 0+a 1 x+a 2 y \\
& Y=b 0+a 2 y+a 1 y
\end{aligned}
$$

where a1 and a2 are the two scale parameters. This model is also known as the four-parameter transformation model and is a special case of the affine model with two constraints (Yaron A Felus, 2006): the scales in both the $\mathrm{x}$ - and y-directions are the same and the two axes are orthogonal. A minimum of two GCPs is required to estimate these four parameters in the similarity model.

Affine transformation model

$$
\begin{aligned}
& X=a 0+a 1 x+a 2 y \\
& Y=b 0+b 1 x+b 2 y
\end{aligned}
$$

where a1, a2, b1, and b2 are the affine parameters. The affine model considers affinity in addition to the translational and rotational bias corrections. A minimum of three GCPs is required to estimate these six parameters in the affine model.

The following process is a geometrical fitting procedure, used to stretch and locally transform the dataset to fit the original shape of the legacy dataset using rubber-sheeting method and constrained least square.

In the rubber sheeting method principal as discussed by Yaron A. Felus (2007), the differences in the $X$ and $Y$ directions are calculated between the boundary corner coordinates in the vector parcel map $\left(x_{i}, y_{i}\right)$ and the surveyed coordinates $(x i$ surveyed, $\left.\mathrm{yi}^{\text {surveyed }}\right)$ as follows:

$$
\begin{aligned}
& X_{\text {dif(i) }}=x_{i}-x_{i}{ }^{\text {surveyed }} \\
& Y_{\text {dif }(i)}=y_{i}-y_{i} \text { surveyed }
\end{aligned}
$$

where $X_{\text {dif(i), }}, Y_{\text {dif(i) }}$ are the differences in the $\mathrm{X}$ and $\mathrm{Y}$ directions on boundary corner $i$. Mathematically, the rubber sheeting technique can be viewed as a surface interpolation procedure performed on the X-differences and on the Y-differences separately, namely:

$$
\tilde{X}_{\operatorname{dif}(p 0)}=\lambda_{1} \cdot X_{\text {dif (1) }}+\lambda_{2} \cdot X_{\text {dif (2) }}+\ldots . \lambda_{n} \cdot X_{\text {dif }(n)}
$$

where $X_{\operatorname{dif}(1)}, X_{\operatorname{dif}(2, \ldots \ldots . .}, X_{\operatorname{dif}(n)}, Y_{\operatorname{dif}(1)}, Y_{\operatorname{dif}(2, \ldots \ldots . .}, Y_{\operatorname{dif}(n)}$ are the $X$ and $Y$ - differences as calculated by equation $(10) ; \lambda_{1}, \lambda_{2}, \ldots, \lambda_{n}$ 
are the interpolation coefficients; and $\tilde{X}_{\operatorname{dif}(p 0)}, \tilde{Y}_{\text {dif (p0) }}$ are the calculated local differences (distortions) which are added to the initial coordinates of point $p_{0}$ (i.e., coordinates from the vector parcel map). Thus, the final coordinates of point $p_{0}$ $\tilde{x}_{p 0}=x_{p 0}-\tilde{X}_{\text {dif }(p 0)}, \tilde{y}_{p 0}=y_{p 0}-\tilde{Y}_{\operatorname{dif}(p 0)}$ where $x_{p o}$ and $y_{p o}$ are the initial coordinates of point $\mathrm{p} 0$ taken from the vector parcel map.

Another method of geometrical fitting is using constraints least square which was introduced by F. R. Helmert in 1872 (Ghilani, 2010). In this procedure, the constraint equation(s) border the reduced normal equations as

$$
\left[\begin{array}{ccc}
A^{\mathrm{T}} W A & \vdots & C^{\mathrm{T}} \\
\cdots & \cdots & \cdots \\
C & \vdots & 0
\end{array}\right]\left[\begin{array}{c}
X_{1} \\
\cdots \\
X_{2}
\end{array}\right]=\left[\begin{array}{c}
A^{\mathrm{T}} W L_{1} \\
\cdots \\
L_{2}
\end{array}\right]
$$

To establish this matrix, the normal matrix (Design Matrix=A, Weightage Matrix $=\mathrm{W}$ and Observation Matrix $=\mathrm{L}$ ), and its matching constants matrix are formed. These observation equations are then included in the normal matrix as additional rows $[C]$ and columns $[C T]$ in Equation (8) and their constants are added to the constants matrix as additional rows [L2] in Equation (8). The inverse of this bordered normal matrix is computed. The matrix solution of the Equation (8) is

$$
\left[\begin{array}{c}
X_{1} \\
\cdots \\
X_{2}
\end{array}\right]=\left[\begin{array}{ccc}
A^{\mathrm{T}} W A & \vdots & C^{\mathrm{T}} \\
\cdots & \cdots & \cdots \\
C & \vdots & 0
\end{array}\right]^{-1}\left[\begin{array}{c}
A^{\mathrm{T}} W L_{1} \\
\cdots \\
L_{2}
\end{array}\right]
$$

The final stage in the workflow involves a quality evaluation. The two level of evaluation based on the parameters of positional accuracy and geometrical fitting will be used in the assessment. Method of point displacement residual test (positional accuracy assessment) and angle/area statistical test (geometrical fitting assessment) will be applied and the final output of this process will be submitted in the accurate cadastral database. Figure 3 describes the entire process of suggested PAI as discussed above.

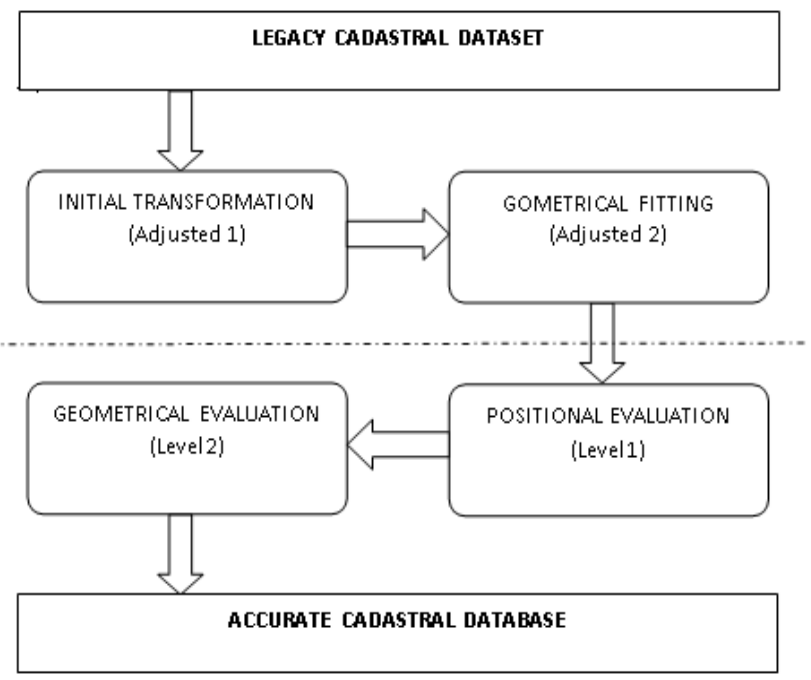

Figure 3. PAI concept

\section{CONCLUSION}

This review study investigated different technical aspects in the process of enhancing legacy datasets for the creation of an accurate coordinate-based cadastre using PAI. A three-step procedure was concluded as suggestion to enhance the accuracy of cadastral maps and a few unique methods were reviewed during this process. A geometric fitting technique should be selected efficiently to locally translate, rotate, and scale the legacy dataset alignment with new measurements. Finally, this review article provides alternative tools for the enhancement of digital cadastral maps. These tools expectantly will assist the DSMM in managing the NDCDB toward implementing a fully accurate digital cadastre by the next decade.

\section{REFERENCES}

Arvanitis, A., \& Koukopoulou, S. (1999). Managing Data During the Update of the Hellenic Cadastre. Paper presented at the FIG Com3 Annual Meeting, Budapest.

Buyong, T., \& Kuhn, W. (1990). Local Adjustment for a Measurement-Based Multipurpose Cadastre Systems. Paper presented at the XIX Congress of FIG.

Casado, M. L. (2006). Some basic mathematical constraints for the geometric conflation problem. Paper presented at the Proceedings of the 7th international symposium on spatial accuracy assessment in natural resources and environmental sciences.

Cobb, M. A., Chung, M. J., Foley III, H., Petry, F. E., Shaw, K. B., \& Miller, H. V. (1998). A rule-based approach for the conflation of attributed vector data. GeoInformatica, 2(1), 7-35. Desa, G. (2005). Social and Economic Impact of Coordinated Cadastral System (CCS) Implementation for Peninsular Malaysia. Melaka, Malaysia.

Donnelly, N., \& Hannah, J. (2006). An Assessment of the Precision of the Observational Data Used in New Zealand's National Cadastral system. Survey Review, 38(300).

Doytsher, Y., \& Gelbman, E. (1995). Rubber-sheeting algorithm for cadastral maps. Journal of surveying engineering, 121(4), 155-162.

Durgin, P. M. (1993). Measurement-Based Databases: One Approach to the Integration of Survey and GIS Cadastral Data. Surveying and Land Information Systems, 53(1), 41-47.

Effenberg, W. W., Enemark, S., \& Williamson, I. P. (1999). Framework for Discussion of Digital Spatial Data Flow within Cadastral Systems. Australian surveyor, 44(1), 35-43.

Felus, Y. A. (2006). On linear transformations of spatial data using the structured total least norm principle. Cartography and Geographic Information Science, 33(3), 195-205.

Felus, Y. A. (2007). On the Positional Enhancement of Digital Cadastral Maps. Survey Review, 39(306), 268-281. doi: 10.1179/175227007x197183 
Fradkin, K., \& Doytsher, Y. (2002). Establishing an urban digital cadastre: analytical reconstruction of parcel boundaries. Computers, Environment and Urban Systems, 26(5), 447-463.

Ghilani, C. D. (2010). Adjustment computations: spatial data analysis: John Wiley \& Sons.

Gielsdorf, F., Gruendig, L., \& Aschoff, B. (2004). Positional accuracy improvement-A necessary tool for updating and integration of GIS data. Paper presented at the Proceedings of the FIG working week.

Greenfeld, J. S. (1997). Least Squares Weighted Coordinate Transformation Formulas and Their Applications. Journal of surveying engineering, 123(4), 147-161.

Hesse, W. J., Benwell, G. L., \& Williamson, I. P. (1990). Optimising, maintaining and updating the spatial accuracy of digital cadastral data bases. Australian surveyor, 35(2), 109119.

Hope, S., Gordini, C., \& Kealy, A. (2008). Positional accuracy improvement: lessons learned from regional Victoria, Australia. Survey Review, 40(307), 29-42. doi: $10.1179 / 003962608 \times 253457$

Hope, S., Kealy, A., \& Hunter, G. (2006). Improving positional accuracy and preserving topology through spatial data fusion. Paper presented at the 7th international symposium on spatial accuracy assessment in natural resources and environmental sciences. International Spatial Accuracy Research Association (ISARA).

Kang, H. (2002). Analytical conflation of spatial data from municipal and federal Government agencies. The Ohio State University.

Klebanov, M., \& Doytsher, Y. (2009). Cadastral Triangulation: A Block Adjustment Approach for Joining Numerous Cadastral Blocks. Nordic journal of surveying and real estate research, 4 . Koch, K.-R. (2013). Parameter estimation and hypothesis testing in linear models: Springer Science \& Business Media.

Merrit, R., \& Masters, E. (1999). Digital cadastral upgradesA progress report. Paper presented at the Proceedings of the First International Symposium on Spatial Data Quality.

Merritt, R. (2005). An assessment of using least squares adjustment to upgrade spatial data in GIS. University of New South Wales.

Mikhail, E. M., \& Ackermann, F. E. (1982). Observations and least squares: Univ Pr of Amer.

Mohd Yusoff, M. Y., \& Abdul Halim, N. Z. (2012). Unleashing the Full Potential of eKadaster on The Cadastral System of Malaysia. Paper presented at the Nineteenth United Nations Regional Cartographic Conference for Asia and the Pacific, Bangkok, Thailand.

Mohd Yusoff, M. Y., Jamil, H., \& Abdul Halim, N. Z. (2013). eKadaster: A Learning Experience for Malaysia. Paper presented at the FIG Pacific Small Island Developing States Symposium Policies and Practices for Responsible Governance, Suva, Fiji.
Morgenstern, D., Prell, K., \& Riemer, H. (1989). Digitisation and Geometrical Improvement of Inhomogeneous Cadastral Maps. Survey Review, 30(234).

Omar, A. H., A Kadir, A. M., \& Sidek, R. M. S. (2006). Development of Automated Cadastral Database Selection and Visualization System to Support the Realization of Modern Cadastre in Malaysia: Faculty of Geoinformation and Real Estate, Universiti Teknologi Malaysia.

Perelmuter, A., \& Steinberg, G. (1992). LIS and Cadastre in Israel. Paper presented at the Proceedings of the Israeli GIS/LIS'92.

Rönsdorf, C. (2008). Positional Accuracy Improvement (PAI) Encyclopedia of GIS (pp. 885-891): Springer.

Saalfeld, A. (1988). Conflation automated map compilation. International Journal of Geographical Information System, 2(3), 217-228.

Saalfeld, A. (1993). Automated map conflation. University of Maryland, Washington DC.

Sisman, Y. (2014). Coordinate transformation of cadastral maps using different adjustment methods. Journal of the Chinese Institute of Engineers, 37(7), 869-882.

Tamin, N. (1995). A Methodology to Create a Digital Cadastral Overlay Through Upgrading Digitized Cadastral Data. Surveying and Land Information Systems, 55(1), 3-12.

Ting, L., \& Williamson, I. P. (1999). Cadastral Trends: A Synthesis. Australian surveyor, 44(1), 46-54.

Tong, X., Liang, D., Xu, G., \& Zhang, S. (2011). Positional Accuracy Improvement: A Comparative Study in Shanghai, China. International Journal of Geographical Information Science, 25(7), 1147-1171.

Tong, X., Shi, W., \& Liu, D. (2005). A least squares-based method for adjusting the boundaries of area objects. Photogrammetric engineering \& remote sensing, 71(2), 189195.

Tong, X., Shi, W., \& Liu, D. (2009). Introducing scale parameters for adjusting area objects in GIS based on least squares and variance component estimation. International Journal of Geographical Information Science, 23(11), 14131432.

Wang, J., Di, K., \& Li, R. (2005). Evaluation and Improvement of Geopositioning Accuracy of IKONOS Stereo Imagery. Journal of surveying engineering, 131(2), 35-42.

Wolf, P., \& Ghilani, C. (2006). Adjustment Computations Spatial Data Analysis: Hoboken. 